\title{
Complementary Passive Hydrotherapy WATSU (WaterShiatsu) in the Rehabilitation of a Severely Traumatized Accident Survivor - a Prospective Case Report
}

Category: Complementary and Traditional Medicine and Therapies

Introduction: The implementation of complementary and alternative therapies into conventional treatment schemes is gaining popularity. However, their use is widely depending on patients' drive. This case-report focuses on a patient's experience of the integration of WATSU (WaterShiatsu) in rehabilitative care.

Patient: A 52 year old woman survived a severe motorcycle-accident in which she sustained several fractures on the right side of her body, including ribs, pelvis, and femur. After discharge from stationary care, she independently added WATSU to her rehabilitative regimen.

Treatment approach: WATSU is a passive form of hydrotherapy in warm water that aims at relaxation, pain relief, and a sense of secureness. In the reported case, an experienced WATSUtherapist who is also trained in physiotherapy and psychosomatics delivered weekly sessions of one hour duration.

Measures used: Qualitative data were collected by patient's diary. Also the therapist's notes including The Patient Specific Functional Scale (PSFS) were considered.

Main outcomes: The patient associated WATSU with trunk mobilization (followed by ameliorated breath), reconciliation with her body, and emotional discharge. She ascribed WATSU lasting effects on her body-image. The therapist employed WATSU for careful mobilization and to equalize awareness throughout the body. The PSFS displayed continuous improvement in all categories except usage of public transportation. Due to complications (elevated inflammation markers) only 6 of 8 scheduled sessions were administered.

Conclusions: WATSU was experienced helpful in approaching conditions that are difficult to address by conventional physiotherapy. In early rehabilitation, additional medical/physiotherapeutic skills of contributing complementary therapists are advocated. 